\title{
Proton Glass Dielectric Susceptibility Compared with Monte Carlo and Bound Charge Semiconductor Model Predictions
}

\author{
Authors: V.H. Schmidt, Z. Trybula, D. He, J.E. \\ Drumheller, C. Stigers, Z. Li, and F.L. Howell
}

This is an Accepted Manuscript of an article published in Ferroelectrics in June 1990, available online: http://www.tandfonline.com/10.1080/00150199008214569.

Schmidt, V. H., Z. Trybula, D. He, J. E. Drumheller, C. Stigers, Z. Li, and F. L. Howell. "Proton Glass Dielectric Susceptibility Compared with Monte Carlo and Bound Charge Semiconductor Model Predictions." Ferroelectrics 106, no. 1 (June 1, 1990): 119-124.

doi: 10.1080/00150199008214569.

Made available through Montana State University's $\underline{\text { ScholarWorks }}$

scholarworks.montana.edu 


\section{PROTON GIASS DIELECTRIC SUSCEPTIBILITY COMPARED WITH MONTE CARIO AND BOUND CHARGE SEMICONDUCTOR MODEL PREDICTIONS}

V.H. SCHMIDT, Z. TRYBUZA, D. HE, J.E. DRUMHELLER, C. STIGERS, Z. LI, and F.L. HOWELL*

Physics Dept., Montana State Univ., Bozeman, MT 59717

*Physics Dept., U. North Dakota, Grand Forks, ND 58202

Abstract our latest results for dielectric permittivity and loss and protonic conductivity in RADP, RADA and DRADA proton glasses are presented. Improvements in our "bound charge semiconductor" model for dielectric behavior are discussed. Monte Carlo studies of the phase diagram and polarization decay are described. Bias order parameter vs. temperature plots from the simulation and from ND, deuteron NMR lineshapes are compared.

We describe here our proton glass dielectric measurements, and their interpretation by Monte Carlo simulations based on a short-range-interaction model and by an analytic model for diffusion of effective charge carriers.

We made dielectric measurements on $50 \%$ ammoniated rubidium dihydrogen phosphate (RADP) between 93 and $348 \mathrm{~K}$. The losses in this temperature range are caused by protonic conductivity, which is plotted in Fig. 1. The conductivity is similar to that of other crystals in this family.

The inverse permittivity plotted in Fig. 2 gives a straight Curie-Weiss plot for $\varepsilon_{\text {- near }} 5$. The Curie-Weiss constant is $2800 \mathrm{~K}$, close to the slater theory value of $\mathrm{a}^{2} \mathrm{CP}_{\mathrm{s}}{ }^{2} / 4 \mathrm{k} \varepsilon_{\mathrm{o}}=2700 \mathrm{~K}$.

Dielectric results are presented also for 208 ammoniated rubidium dihydrogen arsenate (RADA), in Figs. 3 and 4 . These show typical proton glass behavior, similar to our results in 35\% ammoniated RADA. ${ }^{2}$ However, the 48 ammoniated crystal

shows unusual behavior. In Fig. 5 the ferroelectric transition at $80 \mathrm{~K}$ is followed by typical proton glass dispersion below $30 \mathrm{~K}$, as seen also in Fig. 6 and in the loss curves of Fig. 7. We believe that local regions with high $\mathrm{Rb}^{+}$ concentrations are ferroelectric, while $\mathrm{NH}_{4}{ }^{+}-\mathrm{rich}$ regions show proton glass behavior. 


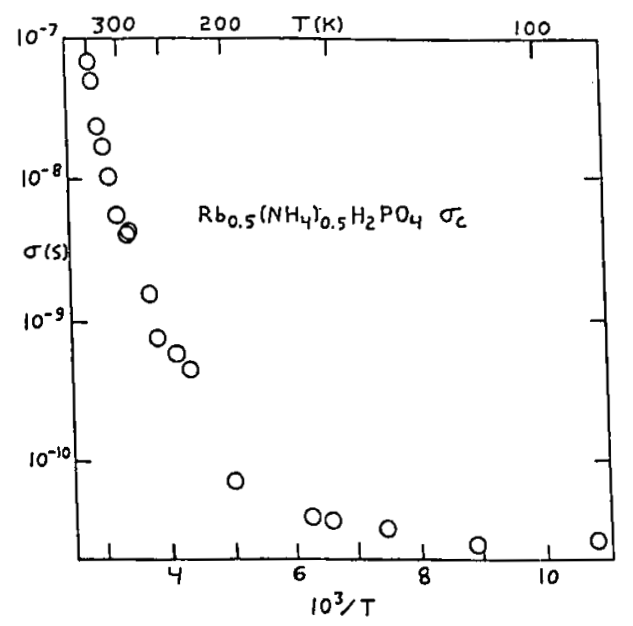

FIGURE 1 Conductivity along $c$ of $50 / 50$ RADP.

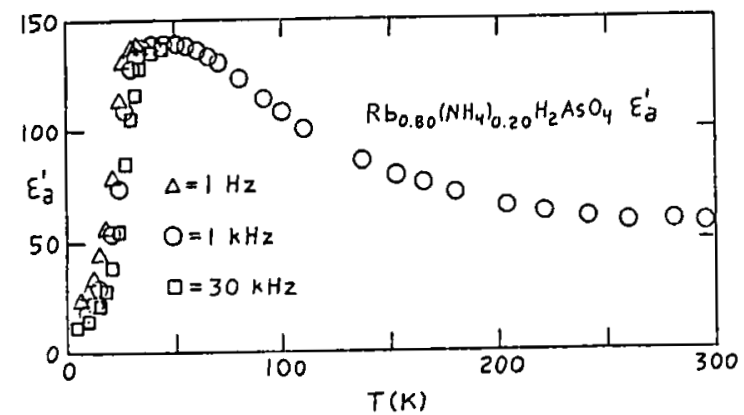

FIGURE 3 Permittivity

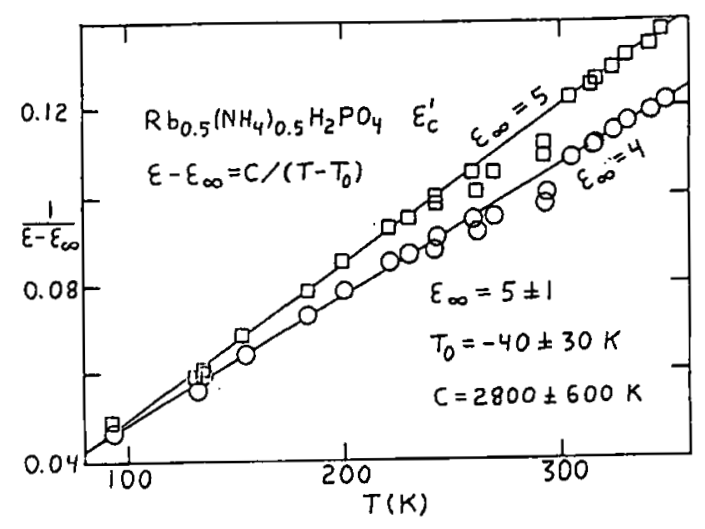

FIGURE 2 Curie-Weiss behavior of 50/50 RADP.

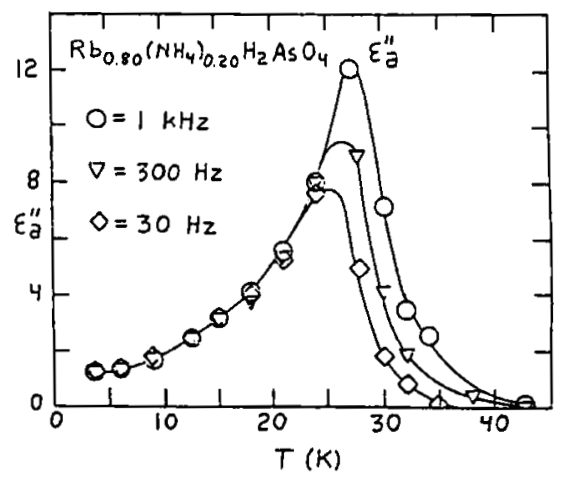

FIGURE 4 Loss along a of $80 / 20$ RADA. along a of $80 / 20$ RADA.

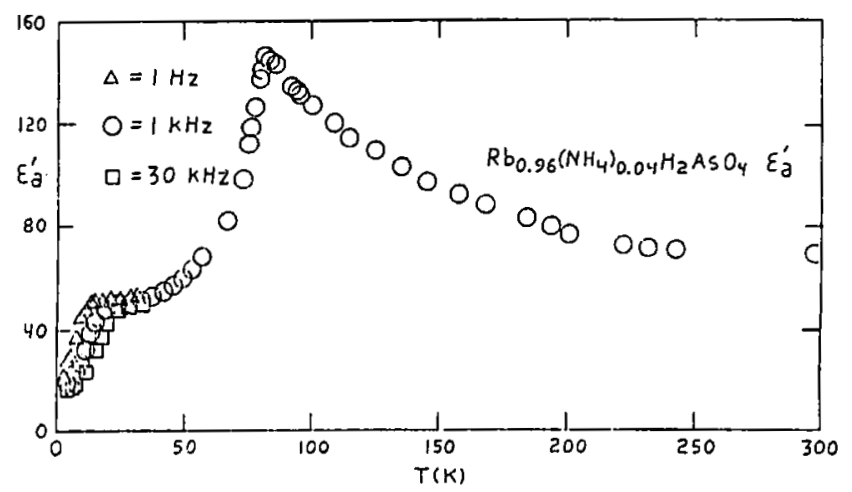

FIGURE 5 Permittivity along a of 96/04 RADA, showing both ferroelectric transition and dielectric dispersion. 


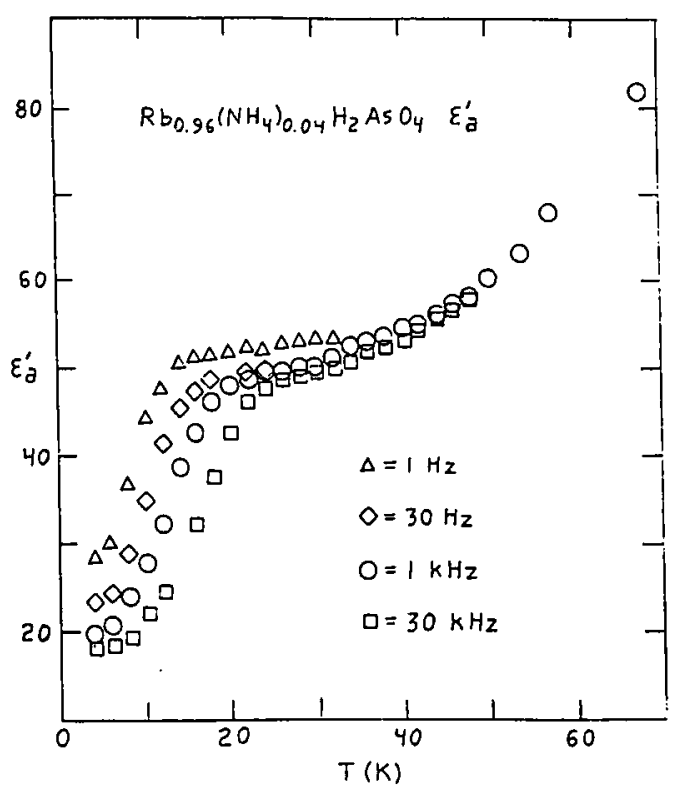

FIGURE 6 Permittivity along a of 96/04 RADA.

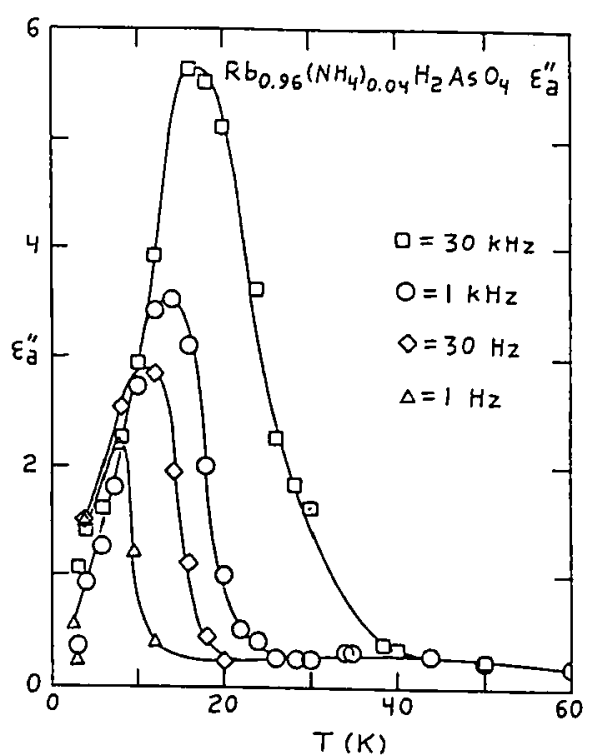

FIGURE $7^{\circ}$ Loss along a of $96 / 04$ RADA.

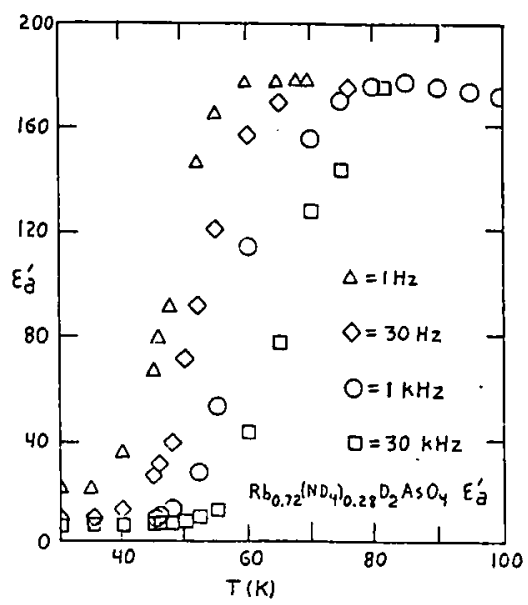

FIGURE 8 Permittivity along a of $72 / 28$ DRADA.

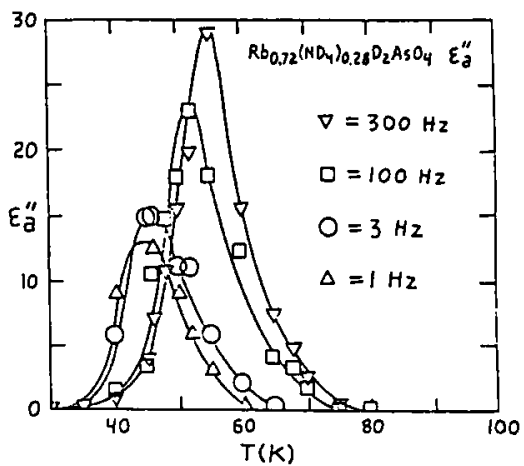

FIGURE 9 Loss along a of $72 / 28$ DRADA. 
We made the first dielectric measurements on deuterated rubidium/ammonium dihydrogen arsenate (DRADA), on a $28 \%$ ammoniated crystal. The results shown in Figs. 8 and 9 resemble those found by courtens ${ }^{3}$ in DRADP, because both DRADA and DRADP show considerable frequency dispersion in this region, while undeuterated RADA and RADP do not.

We are analyzing this dielectric behavior in terms of our "bound charge semiconductor" model 4,5 in which polarization change results from drift of $\mathrm{HPO}_{4}$ and $\mathrm{H}_{3} \mathrm{PO}_{4}$ carriers in an effective field which is the sum of the applied and configurational fields. The relaxation time spread results from mobility being a function of time $t$ after step cutoff of an applied dc field. The polarization decay from initial value $P_{i}$ along the $c$ axis obeys

$$
P / P_{i}=\exp \left[-n\left(\frac{1}{1-q}-\frac{T_{0}}{T}\right)\left(\frac{k T}{E_{d}} \ln \frac{T}{T_{0}}\right)^{4}\right]
$$

where $\mathrm{n}$ is fractional carrier density, $\mathrm{T}_{0}$ is curie-Weiss temperature, $\varepsilon_{d}$ is rms diffusion step energy change, $T_{0}$ is attempt time, and $T$ is $t$ for small $t$ and approaches a fixed value for large $t$. This expression includes the dc susceptibility proposed by sherrington and kirkpatrick, ${ }^{6}$ in which the bias order parameter $q$ is evaluated using the expression derived by Pirc, Tadić, and Blinc. 7

An earlier version of our model 8,9 gave loss peaks vs. temperature which were too broad. In the present model, the power of the logarithm in Eq. (1) is 4 instead of 2 , and in its argument $t$ is replaced by $T$ which becomes constant at large $t$. Both changes narrow the range of mobility as $t$ increases, and should fit experiment better.

The Monte Carlo simulations are based on a model 10,11 employing short-range interactions and random bias. We can simulate polarization decay from a state with initial ferroelectric order (Fig. 10) or antiferroelectric order. To find the Edwards-Anderson order parameter we record a proton configuration, let the system evolve, and find the correlation of the new configuration with the previous one. 


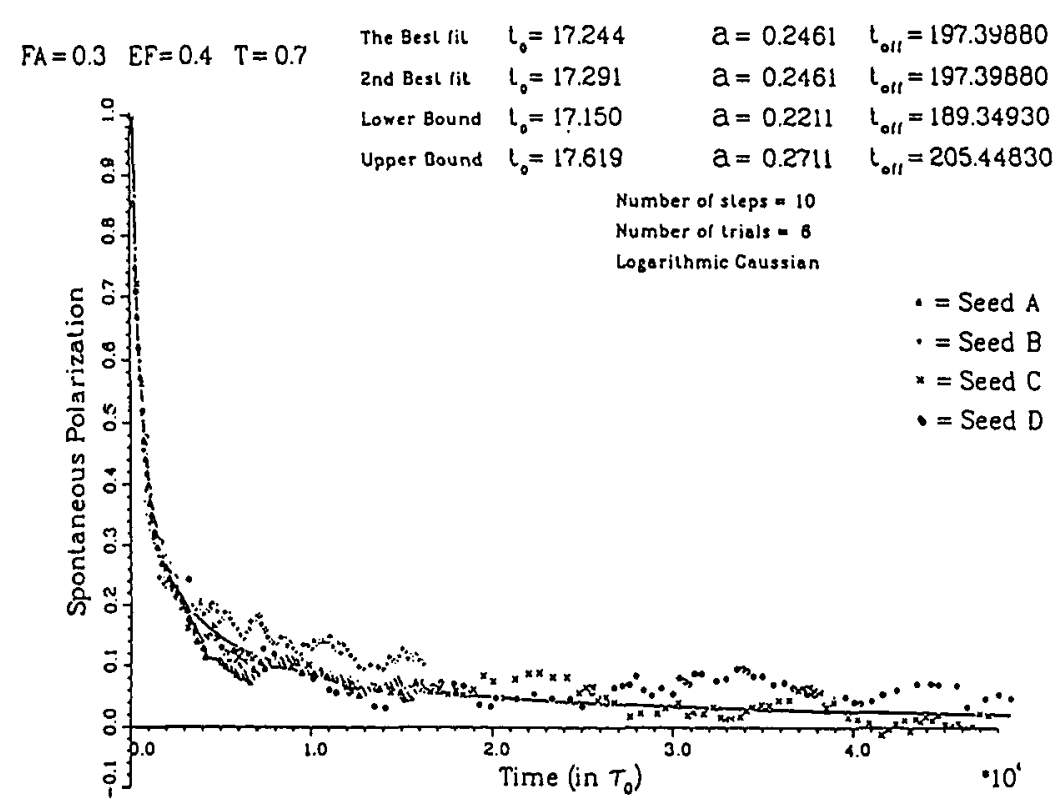

FIGURE 10 Monte Carlo simulation of polarization decay in proton glass, showing logarithmic gaussian best fit to four runs.

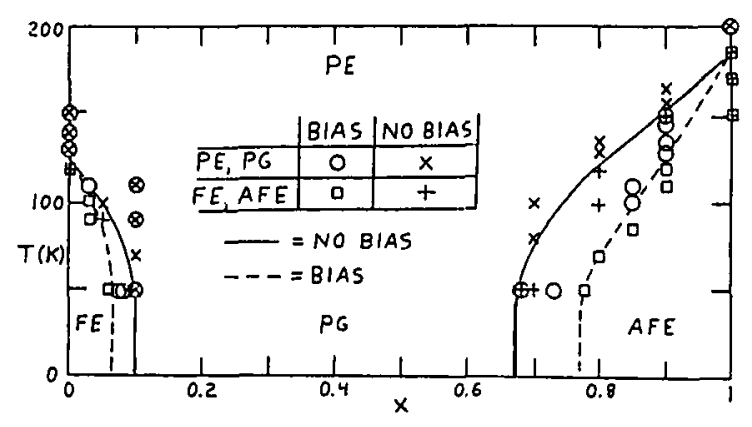

FIGURE 11 Monte Carlo determination of phase diagram for a proton glass model, showing phase boundaries with and without a random bias field.

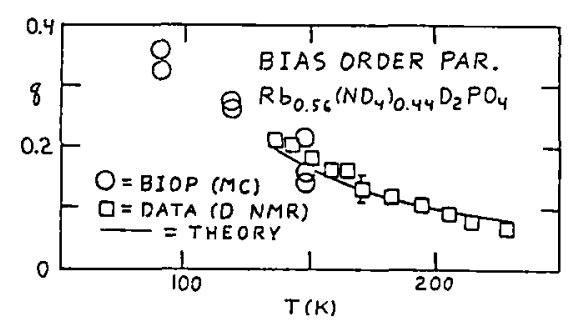

FIGURE 12 Monte Carlo determination of bias order parameter for a proton glass model, compared with values found (Ref. 12) by deuteron NMR and theory for 56/44 DRADP. 
We find the bias order parameter similarly, but warm and then cool the system so it loses memory of the particular potential minimum it may have been trapped in.

To find the phase diagram, we assume the system is in the ferroelectric or antiferroelectric state if it retains $90 \%$ of such initial order after a long running time. We found the phase diagram both with and without random bias, as shown in Fig. 11. The bias widens the proton glass concentration range, as expected, because in the $\mathrm{NaCN} / \mathrm{KCN}$ system which has no frustration, random bias alone produces a phase diagram similar to that of proton glass systems.

We found the bias order parameter vs. temperature from our Monte Carlo simulation and compared it to that found from deuteron NMR of ammonium deuterons in DRADP proton glass, ${ }^{12}$ as shown in Fig. 12. The agreement is quite good.

In summary, we have shown new dielectric results, particularly for arsenate proton glasses. Our Monte Carlo and bound charge carrier models which are based on the crystal structure and interactions explain these results well.

This work was supported by NSF Grant DMR-8714487.

\section{REFERENCES}

1. J. C. Slater, J. Chem. Phys. 9, 16 (1941).

2. Z. Trybula, V. H. Schmidt, J. E. Drumheller, D. He, and

z. Li, Phys. Rev. B 40, 5289 (1989).

3. E. Courtens, Phys. Rev. B 33, 2975 (1986).

4. V. H. Schmidt, Bull. Am. Rhys. Soc. 34, 827 (1989).

5. V.H. Schmidt, Proc. Ramis Conf., Poznan (April 1989).

6. D. Sherrington and S. Kirkpatrick, Phys. Rev. Lett. 35 , 1792 (1975).

7. R. Pirc, B. Tadic, and R. Blinc, Phys. Rev. B 36, 8607 (1987) .

8. V. H. Schmidt, Ferroelectrics 78, 207 (1988).

9. V. H. Schmidt, J. Molec. Struc. 177, 257 (1988).

10. V. H. Schmidt, J. T. Wang, and P. T. Schnackenberg, Jpn. J. Appl. Phys . 24, Suppl. 24-2, 944 (1985).

11. V. H. Schmidt, Ferroelectrics 72, 157 (1987).

12. R. Blinc, J. Dolinsek, V. H. Schmidt, and D. C. Ailion, Europhys. Lett. 6 , 55 (1988). 